\title{
Establishing a Calculation Method for Estimating the Normal Circumference of the Proximal Interphalangeal Finger Joints
}

\author{
Satoru Nakamura1, Daisaku Tokunaga', Ryo Oda1*, Daigo Taniguchi', Shogo Toyama1, \\ Maki Asada1, Hiroyoshi Fujiwara1, Yutaka Kawahito², Takahiro Seno², Toshikazu Kubo' ${ }^{1}$ \\ ${ }^{1}$ Department of Orthopaedics, Graduate School of Medical Science, Kyoto Prefectural University of Medicine, Kyoto, Japan \\ ${ }^{2}$ Department of Inflammation and Immunology, Graduate School of Medical Science, Kyoto Prefectural University of Medicine, \\ Kyoto, Japan \\ Email: ${ }^{*}$ roda@koto.kpu-m.ac.jp
}

How to cite this paper: Nakamura, S., Tokunaga, D., Oda, R., Taniguchi, D., Toyama, S., Asada, M., Fujiwara, H., Kawahito, Y., Seno, T. and Kubo, T. (2017) Establishing a Calculation Method for Estimating the Normal Circumference of the Proximal Interphalangeal Finger Joints. Open Journal of Rheumatology and Autoimmune Diseases, 7, 186-195. https://doi.org/10.4236/ojra.2017.74019

Received: August 1, 2017

Accepted: November 21, 2017

Published: November 24, 2017

Copyright $\odot 2017$ by authors and Scientific Research Publishing Inc. This work is licensed under the Creative Commons Attribution International License (CC BY 4.0).

http://creativecommons.org/licenses/by/4.0/

(c) () Open Access

\begin{abstract}
Objective: To estimate the normal circumference of the interphalangeal (IP) thumb joint and the proximal IP (PIP) joints from the index finger to the little finger using individual physical parameters such as body height and body weight. Methods: The maximum size on the ring gauge was recorded which caused resistance when the IP joint of the thumb or PIP joints of the index to small fingers were passed through the gauge. RG was defined as the ring gauge number with the same diameter as the circumference of the IP thumb joint and PIP finger joints from the index finger to the little finger. There were 300 healthy subjects and 600 hands. Univariate and multivariate analyses were used to analyze the relationships between RG and the parameters of age, sex, height, weight, and dominant hand, and regression equations were derived. Results: Age, sex and body weight were all statistically significant predictive factors of RG in all fingers. R2, the coefficient of determination in the regression equation, was almost 0.7 for all fingers, indicating that a moderate or strong correlation was observed between RG and the regression equation. Conclusions: The circumference of the IP thumb joint and PIP finger joints from the index finger to the little finger can be estimated in healthy individuals using a calculation formula that takes age, sex, body height and body weight into account.
\end{abstract}

\section{Keywords \\ PIP Joint, Circumference, Ring Gauge}

\section{Introduction}

There are numerous disorders that are classified as orthopedic or collagen dis- 
eases that cause swelling of the fingers. These include rheumatoid arthritis (RA), dactylitis associated with spondylarthritis, and osteoarthritis of the fingers. Accurate qualitative and quantitative diagnosis of finger swelling in these disorders is extremely important for differential diagnosis. During the early stages of rheumatoid arthritis in particular, swelling of the fingers is usually the only symptom, and the importance of excluding forms of arthritis other than RA is even mentioned in the new classification criteria published by the American College of Rheumatology and European League Against Rheumatism [1]. However, in current medical treatment settings, when patients who complain of finger swelling are examined on an outpatient basis, it is not uncommon to be unsure of whether swelling was caused by joint disease, or whether the morphology is characteristic of the patient.

For this reason, if there are clinical parameters that enable clinicians to estimate the normal values for finger circumference, it will be possible to determine whether the patient's finger has a greater-than-normal circumference during the initial examination. We believe this would enable clinicians to determine whether or not a disease state is present. In this study, we established a hypothesis to estimate the normal circumference of the interphalangeal (IP) thumb joint and the proximal IP (PIP) joints from the index finger to the little finger using individual physical parameters such as body height and body weight.

\section{Subjects and Methods}

Approval for study RBMR-C-1291-1 was obtained from the local research ethics committee (Graduate School of Medical Science, Kyoto Prefectural University of Medicine, Kyoto, Japan).

Our subjects were a total of 300 healthy volunteers with no history of finger swelling or trauma (119 men, 181 women, age: 19 - 90 years, mean age: 36.7 years) who were examined at the orthopedic outpatient department for diseases associated with PIP finger joint swelling. The only way to make a diagnosis of exclusion between inflammatory disease and traumatismis to do so during history taking. Imaging investigations, such as plain X-rays, are not used for diagnosis. Our subjects had either not yet started working, or engaged in light physical labor. Individuals in professions that involved long-term periods of heavy physical labor were excluded. We measured the IP thumb joint and PIP finger joints from the index finger to the little finger using ring gauges commonly used in Japan (Japan Custom Size standard; ART No. 40610, sizes \#1-30; Meikosha Mfg. Co., Ltd., Nagoya, Japan) (Figure 1). Japan custom size ring gauges are designed such that the diameter increases by $1 / 3 \mathrm{~mm}$ and the internal circumference increases by approximately $1 \mathrm{~mm}$ each time the size increases by one (Table 1). We explained the measurement method to the subjects, then their joint circumferences were measured by the patients themselves, or with the assistance of a nurse or occupational therapist. Ring gauges were used sequentially from largest to smallest in order to ensure the reproducibility of the circumference 

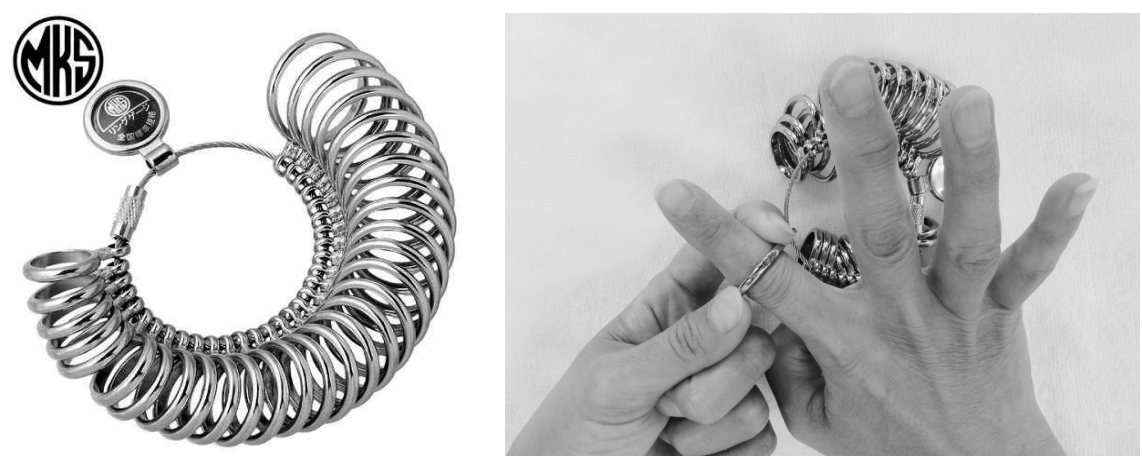

Japan Custom Size standard; ART No. 40610, sizes \#1-30; Meikosha Mfg. Co., Ltd., Nagoya, Japan

Figure 1. Ring gauge (Japan custom size).

Table 1. Table of RG, diameter and internal circumference.

\begin{tabular}{cccccc}
\hline RG & Diameter $(\mathrm{mm})$ & Circumference $(\mathrm{mm})$ & RG & Diameter $(\mathrm{mm})$ & Circumference $(\mathrm{mm})$ \\
\hline 1 & 13 & 40.8 & 16 & 18 & 56.5 \\
2 & $13+1 / 3$ & 41.9 & 17 & $18+1 / 3$ & 57.6 \\
3 & $13+2 / 3$ & 42.9 & 18 & $18+2 / 3$ & 58.6 \\
4 & 14 & 44.0 & 19 & 19 & 59.7 \\
5 & $14+1 / 3$ & 45.0 & 20 & $19+1 / 3$ & 60.7 \\
6 & $14+2 / 3$ & 46.1 & 21 & $19+2 / 3$ & 61.8 \\
7 & 15 & 47.1 & 22 & 20 & 62.8 \\
8 & $15+1 / 3$ & 48.2 & 23 & $20+1 / 3$ & 63.9 \\
9 & $15+2 / 3$ & 49.2 & 24 & $20+2 / 3$ & 64.9 \\
10 & 16 & 50.3 & 25 & 21 & 66.0 \\
11 & $16+1 / 3$ & 51.3 & 26 & $21+1 / 3$ & 67.0 \\
12 & $16+2 / 3$ & 52.4 & 27 & $21+2 / 3$ & 68.1 \\
13 & 17 & 53.4 & 28 & 22 & 69.1 \\
14 & $17+1 / 3$ & 54.5 & 29 & $22+1 / 3$ & 70.2 \\
15 & $17+2 / 3$ & 55.5 & 30 & $22+2 / 3$ & 71.2 \\
\hline
\end{tabular}

measurements. The minimum number of the ring gauge size that allowed the IP thumb joint and PIP finger joints, from the index finger to the little finger, to pass through was recorded. The positions that were actually measured by the ring gauges were the site at which weak resistance occurred (Figure 2(a)), the site at which maximum resistance occurred (Figure 2(b)) and the site at which resistance was attenuated (Figure 2(c)) when the finger was inserted into the ring. We measured the point at which there was maximum resistance, i.e. at Figure 2(b). RG was defined as the ring gauge number with the same diameter as the circumference of the IP thumb joint and PIP finger joints from the index finger to the little finger.

Based on the method described above, we measured the RG from the thumb to the little finger and recorded age, sex, body height, and body weight as 


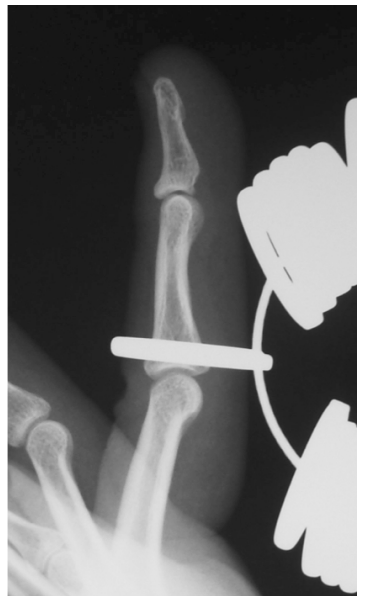

(a)

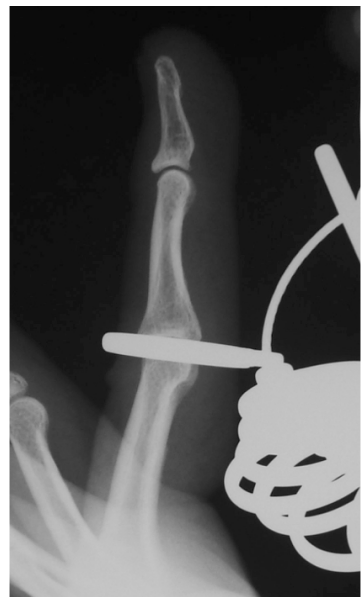

(b)

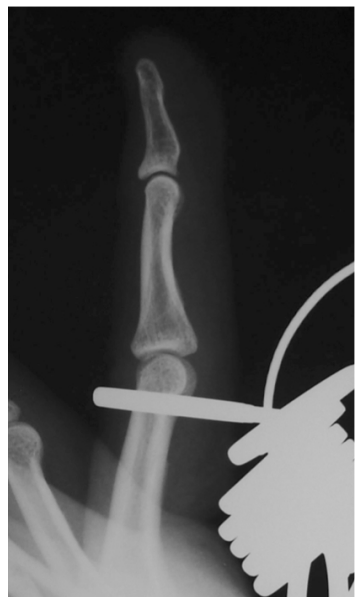

(c)

Figure 2. Measurement position for the ring gauge, (a) Distal end causing resistance; (b) Position at which maximum resistance occurs; (c) Position has passed the proximal part of the PIP.

parameters. Age and sex were confirmed from the patient's medical records, while body height and body weight were measured using a measuring rod and weighing scale in the orthopedic surgery outpatients' department (AD-6228AP, A \& D Co., Ltd., Tokyo, Japan). We conducted regression analysis using the respective finger RGs and each of the parameters, and investigated a method to determine the normal estimated circumference.

We performed statistical analysis using SPSS ver. 23.0 (IBM Japan, Ltd., Tokyo, Japan). The following formula was used to calculate the regression coefficient $R^{2}$ :

$$
R^{2}=1-\left\{\sum_{i=1}^{n}\left(y_{i}-\hat{y}_{i}\right)^{2} /(n-m-1)\right\} /\left\{\sum_{i=1}^{n}\left(y_{i}-\bar{y}_{i}\right)^{2} /(n-1)\right\}
$$

where $y_{i}$ is actual data, $\hat{y}_{i}$ is data estimated from regression equation, $\bar{y}$ is mean value obtained from the whole data, $n$ is the number of data points and $\mathrm{m}$ is the number of independent variables.

During statistical analysis, we performed univariate analysis using the RG and each of the parameters, then performed additional multivariate analysis. A p value less than 0.05 was considered to be statistically significant, and coefficient of determination $R^{2}<0.2$ was defined as no correlation, $0.2 \leq R^{2}<0.4$ was defined as a weak correlation, $0.4 \leq R^{2}<0.7$ was defined as a moderate correlation and $R^{2} \geq 0.7$ was defined as a strong correlation.

During this study, RG was used as a dependent variable, while age, sex, body height, and body weight were used as independent variables, and all were used to perform linear regression analysis. The RG estimation equation was used for analysis.

\section{Results}

The purpose of this study was to construct a highly accurate estimation equation 
using the RG in relation to age, sex, body height, and body weight. It was therefore recommended that the sample size be as large as possible. For this reason, we calculated the test power during a post-hoc power analysis to prove that the 300 subjects who were ultimately studied were statistically adequate. A linear regression model made up of the four variables age, sex, body height, and body weight was defined as significant when the $R^{2} \geq 0.7$, based on an a level of 0.05 . If the variable was significant, then the power of the sample size of 300 subjects who were analyzed during this study was 0.999 . Accordingly, we believe that the sample size in this study was adequate to ensure a power $\geq 0.8$.

The results of univariate analysis of the RG and the parameters age, sex, body height and body weight for healthy fingers are shown in Table 2. Sex, body height and body weight were all statistically significant predictive factors of RG for all fingers. Age was a significant predictive factor of RG in both thumbs and the index finger of the dominant hand, although it was not a significant factor for other fingers. $R^{2}$, the coefficient of determination in the regression equation was below 0.1 for age for all fingers, indicating that no correlation was observed. Body height showed a weak correlation to 9 out of 10 fingers and a strong correlation to 1 finger. Body weight showed a weak correlation to 2 out of 10 fingers and a strong correlation to 8 fingers.

The results of multivariate analysis of the RG and the parameters age, sex, body height and body weight for healthy fingers are shown in Table 3. Age, sex and body weight were all statistically significant predictive factors of RG in all fingers. Although body height was a significant predictive factor of RG in the index finger of the dominant hand and the ring finger in both hands, it was not a significant factor for the other fingers. $R^{2}$, the coefficient of determination in the regression equation, was almost 0.7 for sex in all fingers, indicating that a

Table 2. Results of univariate analysis.

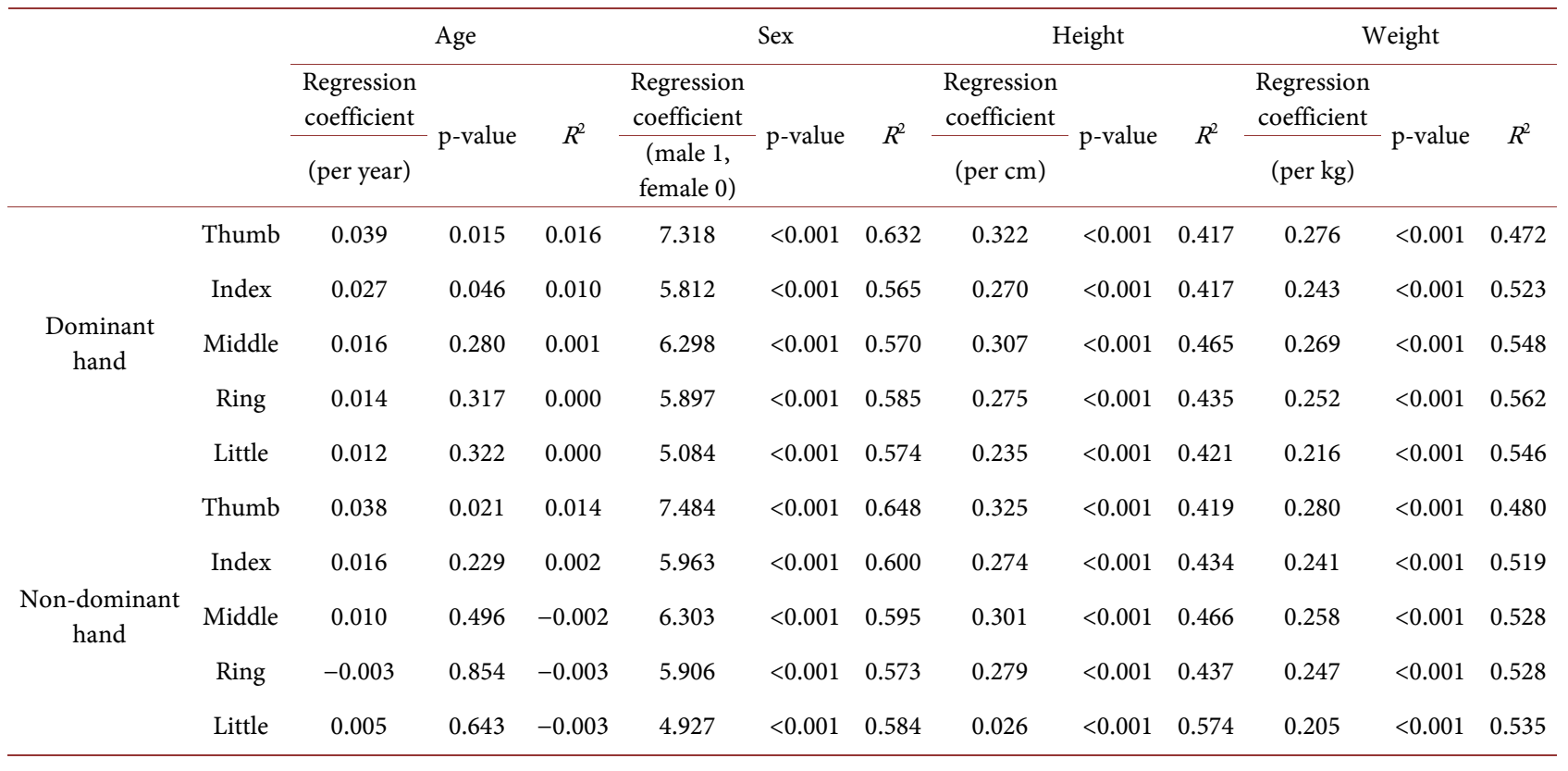


Table 3. Results of univariate analysis.

\begin{tabular}{|c|c|c|c|c|c|c|c|c|c|c|c|}
\hline & & \multirow{3}{*}{ Intercept } & \multicolumn{2}{|c|}{ Age } & \multicolumn{2}{|l|}{ Sex } & \multicolumn{2}{|c|}{ Height } & \multicolumn{2}{|c|}{ Weight } & \multirow{3}{*}{$R^{2}$} \\
\hline & & & $\begin{array}{l}\text { Regression } \\
\text { coefficient }\end{array}$ & \multirow{2}{*}{$\mathrm{p}$-value } & $\begin{array}{l}\text { Regression } \\
\text { coefficient }\end{array}$ & \multirow{2}{*}{$\mathrm{p}$-value } & $\begin{array}{l}\text { Regression } \\
\text { coefficient }\end{array}$ & \multirow{2}{*}{$\mathrm{p}$-value } & $\begin{array}{l}\text { Regression } \\
\text { coefficient }\end{array}$ & \multirow{2}{*}{$\mathrm{p}$-value } & \\
\hline & & & (per year) & & (male 1, female 0 ) & & $($ per $\mathrm{cm})$ & & (per kg) & & \\
\hline \multirow{5}{*}{$\begin{array}{c}\text { Dominant } \\
\text { hand }\end{array}$} & Thumb & -0.318 & 0.071 & $<0.001$ & 5.352 & $<0.001$ & 0.044 & 0.107 & 0.098 & $<0.001$ & 0.720 \\
\hline & Index & -5.636 & 0.054 & $<0.001$ & 3.318 & $<0.001$ & 0.050 & 0.041 & 0.122 & $<0.001$ & 0.686 \\
\hline & Middle & -10.018 & 0.048 & $<0.001$ & 3.129 & $<0.001$ & 0.082 & 0.002 & 0.134 & $<0.001$ & 0.690 \\
\hline & Ring & -5.094 & 0.039 & $<0.001$ & 3.377 & $<0.001$ & 0.033 & 0.172 & 0.136 & $<0.001$ & 0.698 \\
\hline & Little & -8.028 & 0.033 & $<0.001$ & 2.988 & $<0.001$ & 0.025 & 0.252 & 0.116 & $<0.001$ & 0.681 \\
\hline \multirow{5}{*}{$\begin{array}{l}\text { Non-dominant } \\
\text { hand }\end{array}$} & Thumb & 0.119 & 0.068 & $<0.001$ & 5.644 & $<0.001$ & 0.033 & 0.231 & 0.100 & $<0.001$ & 0.732 \\
\hline & Index & -4.040 & 0.042 & $<0.001$ & 3.764 & $<0.001$ & 0.039 & 0.107 & 0.111 & $<0.001$ & 0.689 \\
\hline & Middle & -7.790 & 0.040 & $<0.001$ & 3.591 & $<0.001$ & 0.067 & 0.010 & 0.117 & $<0.001$ & 0.686 \\
\hline & Ring & -4.959 & 0.023 & 0.009 & 3.500 & $<0.001$ & 0.033 & 0.201 & 0.125 & $<0.001$ & 0.640 \\
\hline & Little & -6.480 & 0.025 & $<0.001$ & 3.079 & $<0.001$ & 0.016 & 0.444 & 0.106 & $<0.001$ & 0.672 \\
\hline
\end{tabular}

moderate or strong correlation was observed. We created a program to calculate RGs and circumferences of the IP thumb joint and PIP finger joints from the index finger to the little finger on the following website (Figure 3)

http://www.mito2001.com/sample/furitsu/html/study/rheumatism_cal.html.

\section{Discussion}

The causes of pathological swelling in the fingers may include various elements, such as synovial membrane swelling, hydrarthrosis, flexor tendon tenosynovitis, soft tissue edema, periarticular osteophyte formation, and bone deformation. We believe that these factors may cause finger swelling in isolation or in combination during RA and collagen disorders including spondylarthritis, as well as osteoarthritis. When differentiating between these disorders, it is of course important to perform a qualitative evaluation of this swelling, but before doing so, quantitative determination of whether the finger is swollen, and whether the state is within the normal physiological range, must take place. However, this is frequently not easy.

In previous reports, Helliwell et al. used a special device known as The Leeds Dactylometer to measure the finger circumference in order to diagnose dactylitis that commonly occurs with axial spondylitis, including ankylosing spondylitis (AS) and psoriatic arthritis [2]. The accuracy of the Leeds Dactylometer finger circumference measurements has been evaluated. However, the dactylitis score [3] is calculated by comparison of the circumference of the fingers affected by dactylitis to the circumference of the fingers on the contralateral side. The score also considers the presence of tenderness. Therefore, these measurements are not usually performed due to the difficulty of evaluation in cases with bilateral dactylitis and variability in tenderness. We used ring gauges, which are easier to obtain than the Leeds Dactylometer. 


\begin{tabular}{l|rrrr}
\multicolumn{1}{c}{ age } & 50 years old & sex & male & female \\
\hline height & $160 \mathrm{~cm}$ & height & $45 \mathrm{~kg}$ \\
\hline
\end{tabular}
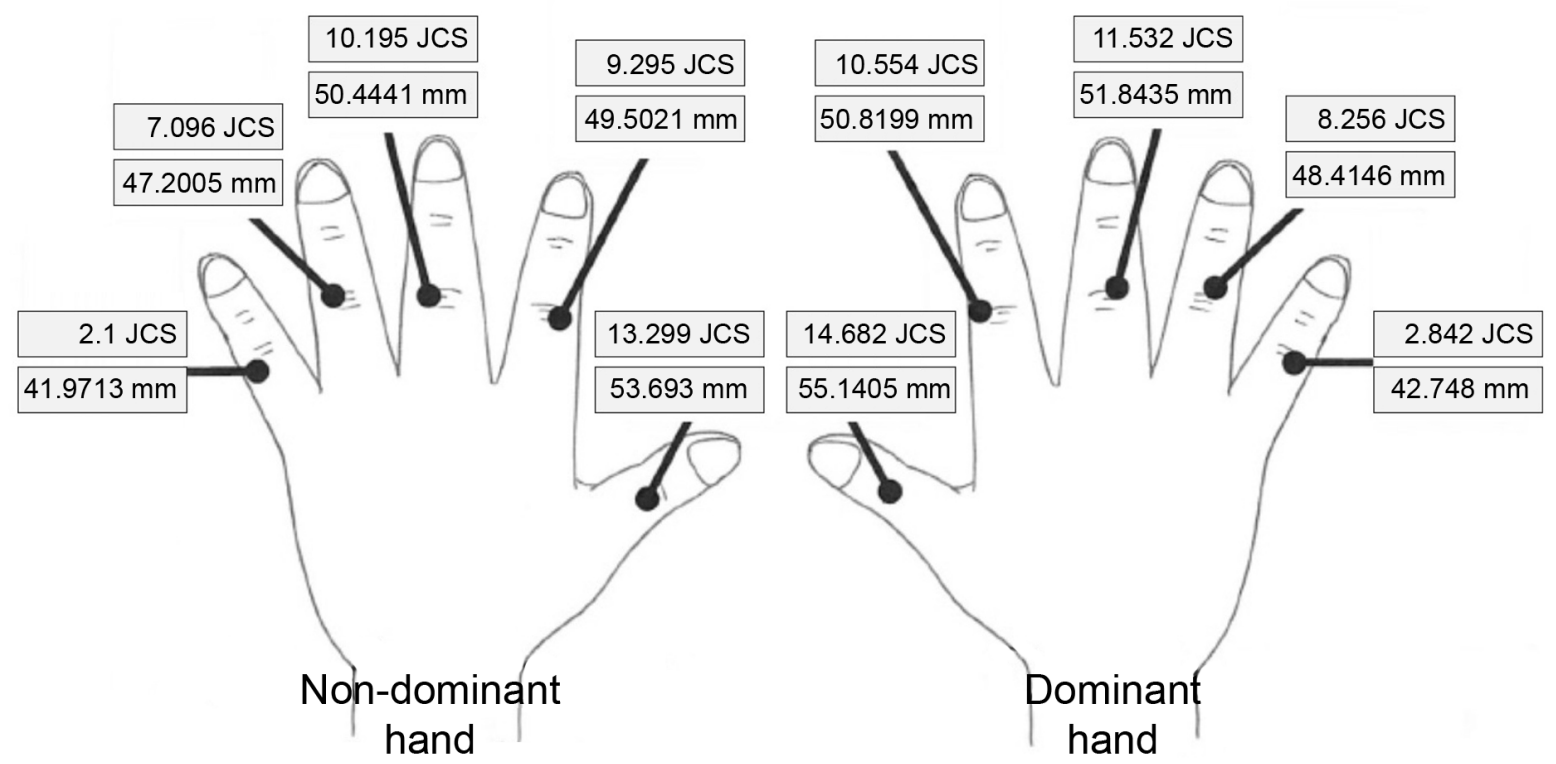

Figure 3. Ring gauge numbers and circumferences of the IP thumb joint and PIP finger joints from the index finger to the little finger, calculated of by age, sex, body height and body weight.

There are various specifications for ring gauges, and each country preferentially uses its own standard. Different specifications are used in the USA, the UK, and Italy, for example. According to the International Organization for Standardization (ISO), ring internal circumference is delimited at $1 \mathrm{~mm}$ intervals from $41 \mathrm{~mm}$ to $76 \mathrm{~mm}$ and ring gauge standardization is progressing, but there is currently no standard.

The Leeds Dactylometer can be used to perform a quantitative evaluation, but when performing actual measurements, the graduations are at intervals of $1 \mathrm{~mm}$, therefore evaluations of $\leq 1 \mathrm{~mm}$ are difficult. The Japan custom size ring gauges are designed such that each increase in size corresponds to an increase of $1 \mathrm{~mm}$ in internal circumference (i.e. external circumference of the joint). For this reason, we believe that it has the same accuracy as the Leeds Dactylometer, albeit semi-quantitative, and has adequate accuracy to be used to measure the circumference of the IP thumb joint and the PIP finger joints, from the index finger to little finger, in clinical practice. The ring gauges can be used to estimate the presence of swelling of the PIP finger joints.

The results of this study show that, when RG numbers are used as objective variables during a univariate analysis, RG is unrelated to age, but related to sex, body height and body weight, although during multivariate analysis, RG was found to be unrelated to body height, but related to age, sex and body weight. 
These differences may be due to the fact that body height and body weight are confounding factors. However, the RG includes not only the skeleton, but also the soft tissues in the joint circumference, therefore we believe that body height and body weight should each be viewed as independent variables and body height should also be used as a parameter in the estimation equation.

During actual medical practice, ultrasound (US) and magnetic resonance imaging (MRI) are used to perform image-based evaluations of finger PIP joint swelling [4] [5] [6].

The greatest advantages of US are its rapid dissemination due to hardware advances and its non-invasiveness. Hyperplasia of the synovial membrane within the joint is not flat and extends proximally and dorsally from the joint space in the PIP finger joints, therefore it is possible to perform accurate examinations using US [7]. Reports state that, compared to the Disease Activity Score for RA (DAS28), the power Doppler (PD) US score exhibits a strong correlation to the progression of joint destruction over time [8]. The frequency of the progression of joint destruction was compared at baseline and after one year in RA patients in clinical remission using examination findings, and MRI and US findings. Examination findings were not able to predict the progression of joint destruction, but US PD was reported to be capable of predicting the progression of joint destruction at an odds ratio of 12.21 [9]. However, disadvantages of US are the fact that considerable time is required to perform US investigations, the high frequency of inter- and intra-observer errors [10], and the differing sensitivity of PD depending on the US device used [11]. The disadvantage of using US devices is the fact that they are qualitative, and lack objectivity. By comparison, the ring gauge is a semiquantitative device, and easy to use, which facilitates easy follow-up examination. Intra- and inter-observer errors were not assessed in this study, and will be a topic for future research.

An advantage of MRI is that it can accurately depict synovial proliferation. Reports state that it is possible to easily diagnose hyperplasia of the finger joint synovial membrane using contrast-enhanced MRI images of the fingers that are taken using gadolinium, with additional processing performed using maximum intensity projection [12] [13]. Changes in synovitis can be easily visualized using contrast-enhanced MRI and it is very useful as a method for presenting patient-friendly results. However, disadvantages of MRI include the fact that contrast-enhancement necessitates intravenous injection of gadolinium, and that the investigations are time-consuming and expensive. In addition, MRI cannot be said to be an international standard investigation method at this point in time. According to the indicators (2013) published by the Organization for Economic Co-operation and Development (OECD), the mean number of MRI machines in 2013 was 14.1 machines per 1 million individuals [14]. In Japan, this number is 46.9 machines per 1 million individuals, meaning that Japan has the highest number of MRI machines, although in several countries, MRI cannot easily be used for diagnosis at present. 
The method described in this paper is not appropriate for differential diagnosis, because it is not capable of providing qualitative diagnosis of finger swelling. However, it is possible to observe changes over time easily using ring gauges due to changes in disease activity and treatment intervention in diseases such as RA that cause changes to the finger PIP joint circumference over a relatively short period. If we observe a relationship when we compare the semi-quantitative evaluation of synovitis using US and MRI to the changes in PIP finger joint circumference using ring gauges, then ring gauges may assist in evaluation of finger PIP joint synovitis and evaluation of the disease state. Furthermore, if ring gauges show some correlation to ultrasound and MRI, so they have the potential to become a powerful medical auxiliary tool in institutions, countries or regions where these tests cannot be easily performed. These will be future research topics.

There were some limitations during this study. The subjects were 300 Japanese individuals, so adequate adjustment for confounding factors using sociodemographic data was not possible. Intra- and inter-observer errors were not assessed for the RG measurements performed during the study. Going forward, studies should perform a comparative investigation of patients with PIP finger joint swelling, based on the results in this study.

\section{Conclusion}

The current study has shown that the circumference of the IP thumb joint and PIP finger joints from the index finger to the little finger can be estimated in healthy individuals using a calculation formula that takes age, sex, body height and body weight into account.

\section{Conflict of Interest}

The authors have no conflict of interest directly relevant to the content of this article.

\section{References}

[1] Aletaha, D., Neogi, T., Silman, A.J., Funovits, J., Felson, D.T., et al. (2010) Rheumatoid Arthritis Classification Criteria: An American College of Rheumatology/European League against Rheumatism collaborative initiative. Arthritis \& Rheumatism, 62, 2569-2581. https://doi.org/10.1002/art.27584

[2] Healy, P.J. and Helliwell, P.S. (2007) Measuring Dactylitis in Clinical Trials: Which Is the Best Instrument to Use? The Journal of Rheumatology, 34, 1302-1306.

[3] Helliwell, P.S., Firth, J., Ibrahim, G.H., Melsom, R.D., Shah, I., et al. (2005) Development of an Assessment Tool for Dactylitis in Patients with Psoriatic Arthritis. The Journal of Rheumatology, 32, 1745-1750.

[4] van der Heijde, D.M., van't Hof, M.A., van Riel, P.L., Theunisse, L.A., Lubberts, E.W., et al. (1990) Judging Disease Activity in Clinical Practice in Rheumatoid Arthritis: First Step in the Development of a Disease Activity Score. Annals of the Rheumatic Diseases, 49, 916-920. https://doi.org/10.1136/ard.49.11.916

[5] Ostergaard, M., Peterfy, C., Conaghan, P., McQueen, F., Bird, P., et al. (2003) 
OMERACT Rheumatoid Arthritis Magnetic Resonance Imaging Studies. Core set of MRI Acquisitions, Joint Pathology Definitions, and the OMERACT RA-MRI Scoring System. The Journal of Rheumatology, 30, 1385-1386.

[6] Szkudlarek, M., Court-Payen, M., Jacobsen, S., Klarlund, M., Thomsen, H.S., et al. (2003) Interobserver Agreement in Ultrasonography of the Finger and Toe Joints in Rheumatoid Arthritis. Arthritis \& Rheumatism, 48, 955-962.

https://doi.org/10.1002/art.10877

[7] Witt, M.N., Mueller, F., Weinert, P., Nigg, A.P., Reindl, C.S., et al. (2014) Ultrasound of Synovitis in Rheumatoid Arthritis: Advantages of the Dorsal over the Palmar Approach to Finger Joints. The Journal of Rheumatology, 41, 422-428. https://doi.org/10.3899/jrheum.131027

[8] Naredo, E., Collado, P., Cruz, A., Palop, M.J., Cabero, F., et al. (2007) Longitudinal Power Doppler Ultrasonographic Assessment of Joint Inflammatory Activity in Early Rheumatoid Arthritis: Predictive Value in Disease Activity and Radiologic Progression. Arthritis \& Rheumatism, 57, 116-124. https://doi.org/10.1002/art.22461

[9] Brown, A.K., Conaghan, P.G., Karim, Z., Quinn, M.A., Ikeda, K., et al. (2008) An Explanation for the Apparent Dissociation between Clinical Remission and Continued Structural Deterioration in Rheumatoid Arthritis. Arthritis \& Rheumatism, 58, 2958-2967. https://doi.org/10.1002/art.23945

[10] Cheung, P.P., Dougados, M. and Gossec, L. (2010) Reliability of Ultrasonography to Detect Synovitis in Rheumatoid Arthritis: A Systematic Literature Review of 35 Studies (1,415 Patients). Arthritis Care \& Research, 62, 323-334. https://doi.org/10.1002/acr.20102

[11] Ten Cate, D.F., Lumie, J.J., van der Ven, M., Hazes, J.M., Kooiman, K., et al. (2013) Very Different Performance of the Power Doppler Modalities of Several Ultrasound Machines Ascertained by a Microvessel Flow Phantom. Arthritis Research \& Therapy, 15, R162. https://doi.org/10.1186/ar4345

[12] Mori, G., Tokunaga, D., Takahashi, K.A., Hojo, T., Fujiwara, H., et al. (2008) Maximum Intensity Projection as a Tool to Diagnose Early Rheumatoid Arthritis. Modern Rheumatology, 18, 247-251. https://doi.org/10.3109/s10165-008-0043-2

[13] Taniguchi, D., Tokunaga, D., Oda, R., Fujiwara, H., Ikeda, T., et al. (2014) Maximum Intensity Projection with Magnetic Resonance Imaging for Evaluating Synovitis of the Hand in Rheumatoid Arthritis: Comparison with Clinical and Ultrasound Findings. Clinical Rheumatology, 33, 911-917. https://doi.org/10.1007/s10067-014-2526-1

[14] OECD Indicators (2015) Health at a Glance 2015. http://www.keepeek.com/Digital-Asset-Management/oecd/social-issues-migrationhealth/health-at-a-glance-2015_health_glance-2015-en\#.WXHuq9PygxM 